indole
treatment
increased the
formation of
persisters
by at least
an order of
magnitude

BACTERIAL PHYSIOLOGY

\title{
From indolence to persistence
}

Bacterial persisters are dormant cells that arise heterogeneously in an antibiotic-susceptible population during entry into stationary phase. These cells are able to tolerate antibiotic treatment and are often involved in chronic and recurrent infections, but the signals that trigger their formation are poorly understood. Writing in Nature Chemical Biology, Vega et al. now show that persistence is induced in part by the stationary phase signalling molecule indole.

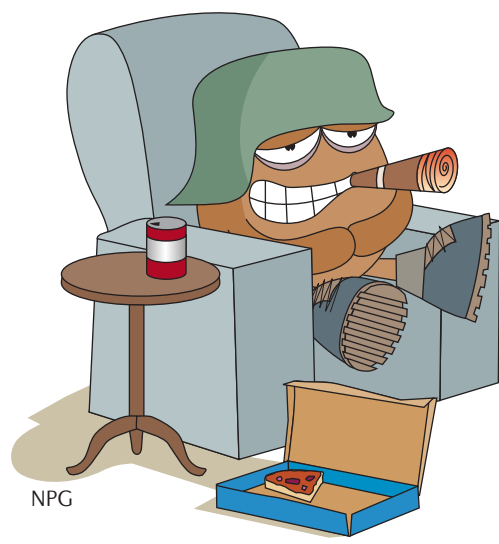

Indole is produced by bacteria under conditions that are known to favour the formation of persisters and has previously been shown to affect membrane and oxidative stress and to increase antibiotic resistance, leading the authors to investigate whether it has a role in persister formation. By treating wild-type Escherichia coli cells with physiological concentrations of indole before adding one of three different bactericidal antibiotics, they found that indole treatment increased the formation of persisters by at least an order of magnitude for each antibiotic. Furthermore, deletion of the tnaA gene, which encodes tryptophanase (the enzyme responsible for catabolizing tryptophan to indole), decreased persister formation by nearly an order of magnitude. However, the tnaA deletion did not completely abolish persister formation, suggesting that additional mechanisms are involved in the induction of persistence. Interestingly, deleting the mtr gene, which encodes the transporter responsible for the uptake of indole into the cytoplasm, led to an increase in persister formation, indicating that the presence of indole in the periplasm or the cell exterior is responsible for the observed effects.

The authors assessed the genomewide transcriptional response to indole in E. coli, finding that indole led to a significant increase in the expression of genes in the OxyR and phage shock pathways, both of which are important for survival in stationary phase cells. Importantly, genetic disruption of either of these two pathways resulted in a substantial reduction in indole-induced persister formation, and disruption of both pathways abolished the phenomenon.

Taken together, these data identify an important role for indole signalling in bacterial persistence and reveal a new avenue for developing therapeutics to combat antibiotic-tolerant bacterial pathogens.

Andrew Jermy

ORIGINAL RESEARCH PAPER Vega, N. M. et al. Signaling-mediated bacterial persister formation. Nature Chem. Biol. 18 Mar 2012 (doi:10.1038/ nchembio.915) 